Artículo de investigación.

Cómo citar: S. Castiblanco, “El juego como estrategia para el fortalecimiento en habilidades cognitivas en alumnos de la Facultad de Ingeniería con pérdida académica”, Inventum, vol. 13, no. 25, pp. 29-40, julio - diciembre, 2018. doi: 10.26620/uniminuto.inventum.13.25.2018. 29-40

Editorial: Corporación Universitaria Minuto de Dios UNIMINUTO.

Recibido: 30 de mayo de 2018 Aceptado: 28 de junio de 2018

Publicado: 3 de septiembre de 2018

Conflicto de intereses: los autores han declarado que no existen intereses en competencia.

\section{EL JUEGO COMO ESTRATEGIA PARA EL FORTALECIMIENTO EN HABILIDADES COGNITIVAS EN ALUMNOS DE LA FACULTAD DE INGENIERÍA CON PÉRDIDA ACADÉMICA}

\author{
GAMES AS A STRATEGY TO REINFORCE \\ COGNITIVE SKILLS IN STUDENTS OF \\ THE SCHOOL OF ENGINEERING WITH \\ ACADEMIC FAILURE \\ O JOGO COMO ESTRATÉGIA PARA \\ FORTALECER AS HABILIDADES \\ COGNITIVAS EM ALUNOS COM PERDA \\ ACADÊMICA DA FACULTADE DE \\ ENGENHARIA
}

Sandra Castiblanco Zerda

\begin{abstract}
Resumen
La pérdida académica por parte de los estudiantes de la Facultad de Ingeniería se da frecuentemente por desatención en la información, inadecuados hábitos de estudio, ausencia en los parciales, falta en la entrega de trabajos y demás detalles que suman a este proceso. Para identificar el fin se implementó una estrategia "basada en el juego" como estrategia para el fortalecimiento en habilidades cognitivas y para el mejoramiento de su rendimiento académico, donde el juego fuese una estrategia para la contribución a su proceso formativo y personal, en los espacios académicos de ingeniería. El diseño de la investigación es de tipo descriptivo, cualitativo; se recolectaron datos mediante sesiones de entrenamiento virtual y presencial, asimismo se entrevistaron a quince estudiantes con pérdida académica de los periodos del segundo semestre del año 2016 y del primer semestre del 2017; posteriormente, se realizó el análisis descriptivo de las sesiones de entrenamiento y las entrevistas, lo que permitió concluir que doce de quince estudiantes cuya participación en las sesiones de entrenamiento tuvo mejor rendimiento académico y fortalecían sus habilidades cognitivas.
\end{abstract}

Palabras clave: Juego, habilidades cognitivas, estudiantes de ingeniería, rendimiento académico.

\footnotetext{
Abstract

Academic failure in students of the School of Engineering usually is the result of a number of factors such as lack of interest in information, inappropriate study skills, non-attendance to midterm evaluations, and failure to submit academic papers. To identify the purpose, the authors implemented a gamebased strategy to further develop cognitive skills and improve academic performance. Games served as an excuse to contribute to the formative and

Sandra Castiblanco Zerda sandra.castiblanco@uniminuto.edu Egresada del programa de Psicología. Corporación Universitaria Minuto de Dios - UNIMINUTO, Colombia 
personal process in engineering courses. This was a descriptive, qualitative research. The collection of data was carried out in on-line and on-site training sessions. The authors interviewed 15 low-achievers who failed academic subjects during the 2016-2 and 2017-1 terms. Then, they performed a descriptive analysis of the training sessions and interviews. They concluded that 12 out of the 15 participants in the training sessions performed higher and improved their cognitive skills.

Keywords: Game, cognitive skills, students of engineering, academic performance.

\section{Resumo}

A perda acadêmica por parte dos estudantes da Faculdade de Engenharia dá-se frequentemente por desatenção na informação, inadequados hábitos de estudo, ausência nos parciais, falta na entrega de trabalhos e demais detalhes que somam a este processo. Para identificar o fim implementou-se uma estratégia "baseada no jogo" como estratégia para o fortalecimento em habilidades cognitivas e para o melhoramento de seu rendimento acadêmico, onde o jogo fosse uma estratégia para a contribuição a seu processo formativo e pessoal, nos espaços acadêmicos de engenharia. 0 desenho da investigação é de tipo descritivo, qualitativo; coletaram-se dados mediante sessões de treinamento virtual e presencial, assim mesmo entrevistaram-se a quinze estudantes com perda acadêmica dos períodos do segundo semestre do ano 2016 e do primeiro semestre do 2017; posteriormente, realizou-se a análise descritiva das sessões de treinamento e as entrevistas, o que permitiu concluir que doze de quinze estudantes cuja participação nas sessões de treinamento teve melhor rendimento acadêmico e fortaleciam suas habilidades cognitivas.

Palavras-chave: Jogo, habilidades cognitivas, estudantes de engenharia, rendimento acadêmico. 


\section{INTRODUCCIÓN}

El juego es una de las primeras actividades asumidas en la infancia, por medio de este se desarrollan competencias y habilidades en torno a la manera de concebir el cuerpo, cómo se comporta este ante estímulos externos en relación con otros, asimismo "el uso de esta estrategia persigue una cantidad de objetivos que están” [1].

Como el juego no es una actividad exclusiva de la infancia, esta actividad es imprescindible para todo el ciclo vital; se tomó como factor importante el identificar si el juego es una estrategia que permite fortalecer habilidades cognitivas y si tiene relación en cuanto al rendimiento académico.

El juego como una estrategia de aprendizaje permite al estudiante fortalecer su resolución de conflictos, enfrentar situaciones y analizar las mismas, además, interiorizar conceptos vistos en el aula, de manera significativa [2].

Si bien dentro de la Corporación Universitaria Minuto de Dios, sede principal, se efectúan actividades lúdicas encaminadas al estudiante, éstas sólo permiten la socialización, la conexión interpersonal y relacional. Este tipo de actividades tienen gran participación y acogida por parte de los estudiantes, sin embargo, se limita a la exploración de otros contextos, es decir, a la consolidación de información proporcionada en las aulas de clase que permite fortalecer competencias y habilidades propias de los programas académicos y la formación personal.

Mediante entrevistas de seguimiento académico aplicadas con los estudiantes en el segundo semestre del año 2016 y primer semestre del año 2017, se evidenció que el bajo rendimiento se vio reflejado por la retención de información, dificultad para prestar atención, olvido en la entrega de trabajos y parciales, dificultades en comprensión de textos. Dado lo anterior, surgió la necesidad de proponer una forma para el fortalecimiento de su aprendizaje, desde una perspectiva apetitiva para los estudiantes.

Teniendo en cuenta el discurso de los estudiantes frente a su rendimiento, se efectuaron sesiones de entrenamiento virtual y presencial teniendo como prevalencia el juego con el fin de fortalecer sus habilidades cognitivas y a su vez mejorar el rendimiento académico en el estudiante.

\section{JUSTIFICACIÓN}

Se ha evidenciado que la Facultad de Ingeniería tiene aproximadamente 3000 estudiantes, número que asciende al pasar de los semestres. Sin embargo, el bajo rendimiento y la pérdida académica por parte de los estudiantes en el segundo semestre del año 2016 es de 413 alumnos, lo que equivale al 13,7\% de los estudiantes; en relación con el primer semestre del año 2017, las pérdidas son de 368 estudiantes inscritos, lo que equivale al $12,2 \%$ de los estudiantes. Según estas cifras, 158 estudiantes han perdido en áreas relacionadas con las ciencias básicas, 209 estudiantes han perdido en áreas relacionadas con procesos lecto-escritores y componentes específicos de los programas académicos.

Teniendo en cuenta lo anterior, aunque las cifras de pérdida han disminuido en un $1,5 \%$, no deja de ser importante proporcionar estrategias y espacios dentro de la universidad para que los estudiantes fortalezcan sus habilidades y competencias dentro de su profesión no solo dándole valor a lo académico, sino también a lo personal.

Dentro de la Corporación Universitaria Minuto de Dios se ha implementado el Modelo de Atención Integral al Estudiante (MAIE) el cual fortalece los procesos académicos, personales y profesionales del estudiante con el fin de propiciar el éxito académico. Este programa realiza seguimiento académico con el objetivo de conocer los factores que afectan el proceso formativo del alumno para brindar orientación pertinente a la situación.

Dicho esto, a partir del seguimiento académico se dio a conocer las razones que causan dificultades en su proceso formativo. Dentro de los seguimientos anteriormente mencionados en situación de reingreso (pérdida académica), reportaron de manera verbal dificultades en las áreas de Ciencias Básicas, falta de concentración en las clases, olvido en las entregas de trabajos, presentación de parciales y dificultades para la comprensión de lectura. Según lo anterior, surgió la necesidad de proponer una forma para el fortalecimiento de su aprendizaje, promoviendo la participación, la creación de espacios de aprendizaje alternativo dentro de la universidad. Se propuso el juego como estrategia para el fortalecimiento de habilidades cognitivas [3], "el objetivo del juego es el afianzar de manera atractiva los conceptos, procedimientos y actividades propuestas 
por el programa". Adicionalmente, proponer una estrategia innovadora, amena y divertida para que el estudiante cambie la percepción de apoyos proporcionados dentro de la universidad.

\section{ANTECEDENTES DEL PROBLEMA}

Una de las preocupaciones constantes de las instituciones de educación superior es la deserción académica y los retos que día a día enfrenta para mitigar las cifras, asimismo este fenómeno permite a las instituciones innovar y diversificar propuestas en la mejora de los programas académicos y la calidad de educación. Las instituciones de educación superior de nuestro país [4], en comparación con otros, deben mejorar no solo sus estructuras, materiales, laboratorios entre otros espacios, sino también las maneras de enseñar y el proponer nuevas o mejores formas para concebir competencias y habilidades para el mundo profesional y laboral.

Teniendo en cuenta lo anterior, para crear este tipo de espacios y mejorar estrategias en la universidad, es importante conocer y reconocer al estudiante no solo por su área académica, sino también resaltar otros aspectos que son imprescindibles para la formación de un profesional, como lo son lo emocional, lo relacional, las habilidades y las competencias por fortalecer dentro y fuera de la institución de educación superior.

Desde el área de Bienestar Universitario se propende identificar las variables por las cuales se tiene pérdida académica y, por ende, la deserción. En los encuentros entre los profesionales de Bienestar Universitario y estudiantes se evidencia día a día que factores cómo el económico, social, la adaptación al contexto universitario, aspectos vocacionales y profesionales, salud mental y física, hábitos de estudio, entre otros, han sido claves para conformar estrategias, con ello se han precisado y se han ejecutado programas para fortalecer y mejorar la calidad de los estudiantes y así permitir su permanencia en la institución de educación superior.

Aunque los apoyos de Bienestar Universitario están dirigidos a los estudiantes, algunos de ellos como el seguimiento académico por pérdida de asignaturas no impacta significativamente a la población, ya que se ve reflejado como un requisito u obligación, mas no para su formación profesional y personal, esto se ha evidenciado durante el primer contacto con el estudiante, cuando muestra desinterés, desagrado y baja motivación frente al proceso.

Por tal motivo, es importante como primera instancia en los estudiantes la necesidad de tomar los apoyos brindados por Bienestar Universitario el crecimiento en su proceso personal y profesional. Asimismo, reconocer formas creativas, llamativas para la población facilitando la extrapolación de los talleres o apoyos a otras áreas de ajuste, no solo la académica.

Al respecto, el juego se comprende como la actividad que se realiza para la entretención o diversión en la que se ejercita, desarrolla o fortalece determinada habilidad [5]. De este modo, el juego es la acción de ejercitar una habilidad de forma entretenida, amena, agradable la cual posibilita el fortalecimiento de habilidades cognitivas y práxicas de la persona.

El juego se estructura como un juego reglado que incluye momentos de acción pre reflexiva y de simbolización o apropiación abstractalógica de lo vivido para el logro de objetivos de enseñanza curriculares [...] cuyo objetivo último es la apropiación por parte del jugador, de contenidos fomentando el desarrollo de la creatividad [6].

El juego es una estrategia que puede ser utilizada para mejorar las metodologías de enseñanza, lo que permite al estudiante mejorar significativamente en su proceso académico, ya que se fortalecen áreas de resolución de conflictos, análisis de contexto entre otras [7].

Se ha determinado otra propuesta basada en juegos, la cual determina que el aprendizaje basado en juegos, lo que determina que el juego y el aprendizaje se fusionen y para apoyar y mejorar la enseñanza en cuanto a las metodologías tradicionales [8]. Este tiene una particularidad y es que también se integra la tecnología lo cual es relevante para la investigación ya que se introduce la misma como medio de ejecución y al análisis.

\section{REFERENTES TEÓRICOS}

Es pertinente conocer que las habilidades cognitivas tienen un rol fundamental en el rendimiento académico porque estas median en el proceso de enseñanza aprendizaje, en la adquisición de conocimiento y en la extrapolación del conocimiento a los contextos inmediatos de los aprendices [9]. 
Asimismo, para el Modelo de Atención Integral al Estudiante (M.A.I.E), es imprescindible proponer estrategias que mejoren dichos procesos de adquisición, dado que en la educación superior el buen rendimiento académico se ve reflejada en calificaciones y estas pueden afectarse en las distintas áreas que complementan el proceso académico.

El rendimiento académico es la suma de diferentes y de complejos factores que actúan en la persona que aprende y ha sido definido con un valor atribuido al logro del estudiante en las tareas académicas. Se mide mediante las calificaciones obtenidas, con una valoración cuantitativa, cuyos resultados muestran las materias aprobadas y reprobadas.

Según lo anterior, es necesario replantear si el rendimiento académico es directamente proporcional a una nota cuantitativa o si hay otros factores que permiten que el estudiante tenga o no rendimiento académico sobresaliente.

Cabe recalcar que las habilidades cognitivas pueden desarrollar o perfeccionarse de una forma dinámica y entretenida, permite la consolidación de la información impartida en el aula de clase, es por eso, que el juego facilita el entrenamiento de habilidades de manera interactiva [10].

El juego es la actividad que comprende elementos externos e internos del sujeto, además, es un mecanismo de ajuste al medio donde está inmerso. Para el fortalecimiento de las habilidades cognitivas con relación al buen rendimiento académico es importante la participación activa del estudiante con el propósito de potenciar las habilidades y así poder extrapolarlas no solo al contexto académico, sino también en su diario vivir.

Desde luego, el juego es una actividad preponderante en la formación de nuestro comportamiento, y también lo es en la educación [11].

El juego es el vehículo más tradicional para la educación. Son la tecnología educativa original, la natural, la que ha recibido el sello de aprobación que ofrece la selección natural. No vemos a los leones hembra impartiendo clase a sus cachorros en la pizarra, ni a los leones adultos escribiendo sus memorias para la posteridad.

Se define la gamificación como el incorporar metáforas de la vida cotiana desde el juego lo que permite el fortalecimiento y el mejoramiento del comportamiento y la motivación [10]. Desde este punto de vista, se pretende fortalecer las habilidades cognitivas con elementos esenciales del juego, lo cual permite que el estudiante sea partícipe activo de su proceso formativo y se integre de manera significativa al área de Bienestar Universitario.

Si se articula el juego con el fortalecimiento de habilidades cognitivas para el mejoramiento del rendimiento académico, se permite conseguir un proceso formativo llamativo, innovador para el estudiante, esto conlleva a la extrapolación de conocimientos en varios contextos.

Teniendo en cuenta las razones por las cuales los estudiantes de Ingeniería tienen bajo rendimiento, esta investigación se puntualizó en habilidades cognitivas como atención, memoria, observación, razonamiento lógico, comprendiéndolas de la siguiente manera:

\section{Atención}

Es la posesión voluntaria de la mente para conocer de forma clara y precisa uno o varios estímulos; nos centraremos en dos tipos de atención selectiva y dividida. En la atención selectiva la función es seleccionar un aspecto del estímulo al cual responder, pasando por alto aquellos aspectos irrelevantes del mismo. La atención dividida comprende que el individuo responde a dos o más actividades al mismo tiempo [12].

\section{Observación}

La observación es la habilidad de mirar a algo o a alguien con detenimiento para adquirir un conocimiento determinado. El aprendizaje por observación se facilita cuando se adquieren y se mejoran las capacidades de observación selectiva, codificación de la memoria y la coordinación de los sistemas sensorio motores [14].

\section{Memoria}

Es un sistema de recolección y almacenamiento de la información de forma involuntaria. La memoria cumple como proceso tres funciones primordiales: recolección de información novedosa, organización de la información de forma lógica y coherente y recuperación de la misma [9].

\section{Razonamiento lógico}

Es la habilidad cognitiva que permite resolver problemas, inferir y aprender de manera consciente, estableciendo relaciones causales y lógicas de los acontecimientos diarios [13]. 
Partiendo de los referentes teóricos, se han propuesto entrenamientos en habilidades en dos modalidades presencial y virtual. En la presencialidad, se tuvo en cuenta materiales como juegos de mesa, disponibilidad de los estudiantes y habilidad a entrenar. En las sesiones virtuales se tuvo en cuenta niveles de dificultad, medios tecnológicos que permitiera el juego como principal estrategia.

\section{FORMULACIÓN DEL PROBLEMA}

Teniendo en cuenta el acompañamiento brindado por M.A.I.E, y según al discurso de los estudiantes en el seguimiento académico, surge la pregunta: ¿es el juego una estrategia que aporta al fortalecimiento de habilidades cognitivas para mejorar el rendimiento académico de los estudiantes con pérdida académica de la Facultad de ingeniería?

\section{METODOLOGÍA}

El tipo de investigación que se empleó es de tipo cualitativo, el cual permite presentar datos y productos descriptivos y el investigador es el sujeto central que recolecta y analiza datos, lo cual exige de él una actitud receptiva y adaptable para construir conocimiento de la observación y la comprensión intuitiva lograda por su permanencia en el campo de acción en donde se encuentra. De igual forma, las palabras, las descripciones, las experiencias son usadas para expresar lo que el investigador ha aprendido acerca de un fenómeno que desea comunicar [16].

En cuanto al análisis e interpretación de la información, esta fue sometida a un estudio sistemático desde el análisis deductivo, lo que permitió la interpretación de datos en función de los objetivos planteados en la investigación.

\section{Población y muestra}

Esta investigación se llevó a cabo en la Corporación Universitaria Minuto de Dios, sede principal, Bogotá, específicamente en la Facultad de Ingeniería. Teniendo en cuenta la magnitud de estudiantes, el muestreo fue por conveniencia, se eligieron 15 estudiantes equivalentes al $10 \%$ de los estudiantes con pérdida académica.
Se precisó que los participantes en la investigación tienen pérdida académica, no se tuvo en cuenta el sexo ya que la muestra fue aleatoria y por conveniencia, las edades de los participantes oscilan entre los 18 y 30 años, los estudiantes participantes están adscritos a los siguientes programas:

- Ingeniería de Sistemas.

- Ingeniería Civil.

- Ingeniería Industrial.

- Tecnología en Redes

- Tecnología en Electrónica.

- Tecnología en Informática.

\section{Instrumentos}

Los instrumentos utilizados para esta investigación fueron los siguientes:

- Entrevistas semiestructuradas: La entrevista es un medio eficaz para la recolección de datos, ya que se obtiene información completa. La entrevista semiestructurada permite tener una presentación simple y libre del tema a indagar [17]:

En el ámbito de un tema determinado, el entrevistador puede plantear la conversación de la forma que desee, plantear las preguntas que considere oportunas y hacerlo en los términos que le parezcan convenientes, explicar su significado, pedir al entrevistado que le aclare algo que no entiende o que profundice sobre algún aspecto cuando lo estime necesario, y establecer un estilo propio y personal de conversación.

- Formularios Google: Esta es una aplicación novedosa que recopila y organiza información según las necesidades del usuario, esta herramienta posibilita analizar datos ya que se encuentra anclada a Excel.

- Juegos de mesa: Los juegos seleccionados para esta investigación fueron los siguientes:

* Jenga

* Damas chinas.

* Acertijos.

* Memoria.

* Encuentra las diferencias.

- Kahoot: Aplicación tecnológica que facilita crear juegos y adaptarlos según las necesidades del usuario, este brinda la conexión entre usuarios por medio de la aplicación y así permite jugar de manera sincronizada en aulas. 


\section{RESULTADOS Y DISCUSIÓN EN RELACIÓN CON LAS ENTREVISTAS DE SEGUIMIENTO}

Las entrevistas efectuadas tuvieron una duración aproximada de 30 minutos. A partir de ellas, se indagó la modalidad de encuentro a las sesiones (presencial o virtual), esto permitió mitigar la variable no asistencia por motivos ajenos a la investigación (falta de tiempo, olvido, clases). Asimismo, fueron tenidas en cuenta las consideraciones éticas con cada participante explicando el objetivo de la misma y su anonimato.

Ahora bien, en las sesiones virtuales aplicadas en formularios de Google, se tenía control de quién participaba de las mismas, el número de aciertos y desaciertos a los juegos; de igual manera, terminada la sesión de entrenamiento se retroalimentaba al participante y también se motivaba a continuar con la siguiente sesión. En la figura 1. Se observa una de las sesiones.

Figura 1. Sesión de entrenamiento virtual I. En la parte superior se define la habilidad por fortalecer, seguido del juego y sus opciones de respuesta. La valoración de esta sesión es de un punto

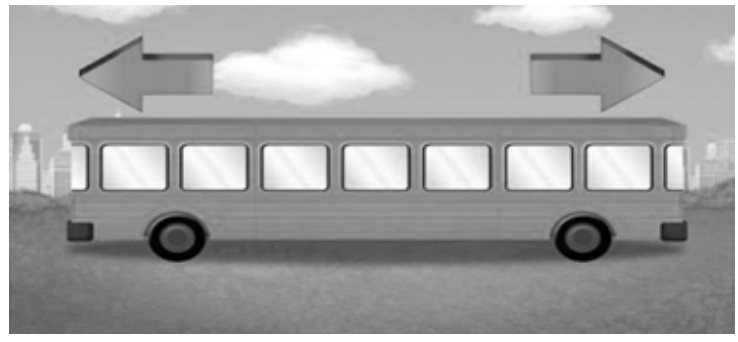

¿En qué dirección va el bus?

$\square$ Derecha

$\square$ Izquierda

$\square$ Ambas

Fuente: Elaboración propia.

En cuanto a los medios tecnológicos ejecutados para las sesiones de entrenamiento presencial se utilizó el Kahoot. Para participar, cada estudiante desde su celular descargaba la aplicación. La actividad consistía en proyectar desde la sala, la plataforma del juego, el estudiante se conectaba desde un pin para activar el juego; ya ingresado el pin, generaba el nombre de usuario, el cual era el número del carné estudiantil. Los juegos tenían un límite de tiempo, así que se podía divisar la agilidad mental y la competencia sana con sus compañeros. En la figura 2 se muestra la plataforma.
Figura 2. Sesión de entrenamiento presencial-lógica. El Kahoot nos permitió observar el tiempo de respuesta. Desde el celular el participante respondía a las preguntas de este divertido acertijo

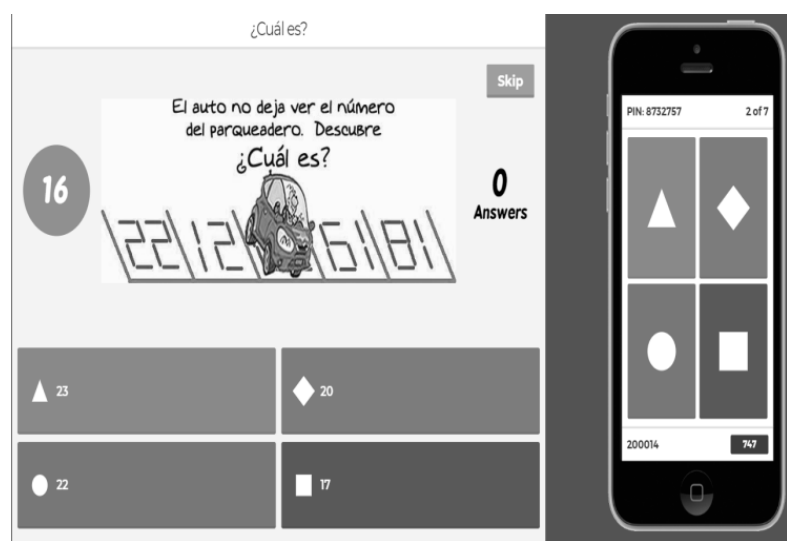

Fuente: Elaboración propia.

En relación con las sesiones de entrenamiento presencial, el cronograma de actividades variaba en cuanto a las habilidades a fortalecer; en la primera parte del encuentro se aplicó un ejercicio rompe hielo y la contextualización de la sesión, en la segunda parte de la sesión en grupos jugaron, jenga, damas chinas, stop numérico entre otros juegos, a medida que avanzaba el juego se cuestionaba la habilidad a fortalecer, en la tercera parte se jugaba con el Kahoot y para el cierre, se retroalimentaba al participante.

Los estudiantes que participaron de la modalidad presencial mostraron disposición a los diferentes juegos; asimismo, mencionaron que la interacción con los compañeros facilitaba la comunicación y se presentaba participación activa frente a las preguntas formuladas. También se propició la competitividad sana y autoevaluación en cuanto a las habilidades que se debían fortalecerse.

En relación con las sesiones virtuales, pudo determinarse que la participación fue mayor, ya que se presentó mayor facilidad de respuesta dado que estaban abiertas en cualquier hora del día. Los estudiantes que participaron de las sesiones mencionaron que son útiles porque visibilizan con claridad la habilidad que se debía fortalecer. Los participantes mencionaron que son didácticas y a la vez entretenidas.

Cabe destacar que dada la participación de los estudiantes a las sesiones tanto presenciales como virtuales 
se crearon sesiones "experto", con un nivel de complejidad mayor en relación con las sesiones anteriores.

En la figura 3 se muestra la comparación entre la participación de sesiones presenciales y sesiones virtuales:

Figura 3. Participación en sesiones de entrenamiento presencial y virtual a partir de datos arrojados en lista de asistencia Isolution y base de datos Excel- formato Google

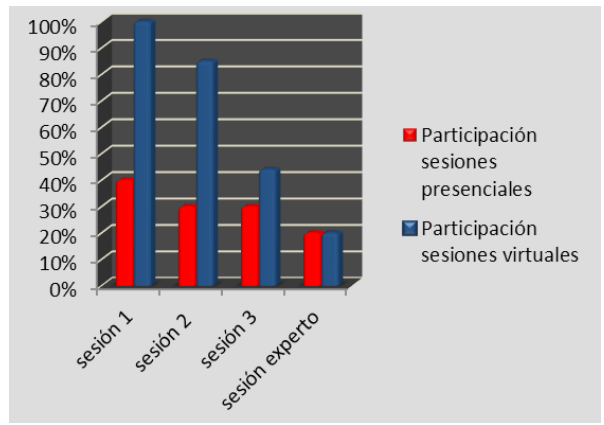

Fuente: Elaboración propia.

Luego de la participación de los quince estudiantes en las sesiones de entrenamiento, se citaron para la aplicación de una entrevista individual con una duración aproximada de 30 minutos, con el objetivo de conocer el impacto de estas sesiones, frente a su rendimiento académico y su área académica, para estas se tuvo en cuenta el ponderado académico antes y después de realizar las sesiones.

Tabla I. Entrevistas individuales. Percepción de participante, frente a las sesiones de entrenamiento en habilidades

\begin{tabular}{|c|c|c|}
\hline PARTICIPANTES & $\begin{array}{l}\text { RESPUESTA } \\
\text { EVALUACIÓN }\end{array}$ & DESCRIPCIÓN \\
\hline Estudiante 1 & “Sí" & $\begin{array}{l}\text { "Hay juegos de memoria y de } \\
\text { concentración en donde tú } \\
\text { tienes que analizar para llegar } \\
\text { a un objetivo, entonces este } \\
\text { me ha ayudado, ya que trato } \\
\text { concentrarme más en mis } \\
\text { actividades sin distraerme". }\end{array}$ \\
\hline Estudiante 2 & "Sí" & $\begin{array}{l}\text { "Considero que las sesiones } \\
\text { sí aportaron en mi proceso } \\
\text { académico, porque me } \\
\text { ayudaron a mejorar la } \\
\text { concentración y la agilidad } \\
\text { en la solución de problemas } \\
\text { matemáticos, y en la } \\
\text { comprensión de los textos". }\end{array}$ \\
\hline Estudiante 3 & “Sí" & $\begin{array}{l}\text { "Ayuda a encontrar las } \\
\text { fortalezas y debilidades, ya } \\
\text { sea de memoria, observación, } \\
\text { atención entre otras". }\end{array}$ \\
\hline
\end{tabular}

\begin{tabular}{|c|c|c|}
\hline PARTICIPANTES & $\begin{array}{l}\text { RESPUESTA } \\
\text { EVALUACIÓN }\end{array}$ & DESCRIPCIÓN \\
\hline Estudiante 4 & “Sí" & $\begin{array}{l}\text { "Considero que me aportan en } \\
\text { el proceso académico, aunque } \\
\text { algunos puntos de los talleres } \\
\text { se vuelven tediosos por el } \\
\text { tiempo que me ha llevado } \\
\text { completarlos, considero que } \\
\text { mi rendimiento ha mejorado } \\
\text { y sería bueno que hubieran } \\
\text { dos o tres talleres más } \\
\text { dinámicos". }\end{array}$ \\
\hline Estudiante 5 & "Sí" & $\begin{array}{l}\text { "Si ha aportado a mi proceso } \\
\text { académico, ya que he } \\
\text { mejorado en la atención y } \\
\text { concentración en la clase". }\end{array}$ \\
\hline Estudiante 6 & “Sí" & $\begin{array}{l}\text { "Son útiles ya que me } \\
\text { hacen entender que tengo } \\
\text { falencias en algunos aspectos, } \\
\text { trabajaré en ellos para } \\
\text { mejorar mi vida profesional y } \\
\text { personal. Gracias". }\end{array}$ \\
\hline Estudiante 7 & “No" & $\begin{array}{l}\text { "No logran captar mi } \\
\text { atención y por tal motivo } \\
\text { me parece aburrido, si fuera } \\
\text { más interactivo tal vez, no } \\
\text { mentira, también me cuesta } \\
\text { mantener la atención en } \\
\text { otras actividades. Creo que es } \\
\text { déficit de atención". }\end{array}$ \\
\hline Estudiante 8 & “Sí" & $\begin{array}{l}\text { "Son demasiado útiles, ya } \\
\text { que mediante esto podemos } \\
\text { tener un mejor desarrollo } \\
\text { mental y la capacidad de } \\
\text { lograr comprender las cosas } \\
\text { sin ningún afán". }\end{array}$ \\
\hline Estudiante 9 & “Sí" & $\begin{array}{l}\text { "Me ayudan con el proceso } \\
\text { mental para la finalidad de } \\
\text { encontrar las estrategias } \\
\text { necesarias para nuestro } \\
\text { desarrollo académico" }\end{array}$ \\
\hline Estudiante 10 & “Sí" & $\begin{array}{l}\text { "Pues la sesiones de } \\
\text { entrenamiento fueron muy } \\
\text { divertidas, ayudaron en lo } \\
\text { que concentración y memoria } \\
\text { se refiere". }\end{array}$ \\
\hline Estudiante 11 & "No" & $\begin{array}{l}\text { "La verdad no sé si son } \\
\text { útiles, más o menos. De } \\
\text { alguna manera ayuda a } \\
\text { mejorar la atención, pero no } \\
\text { certifica que uno tenga mala } \\
\text { atención." }\end{array}$ \\
\hline Estudiante 12 & “Sí" & $\begin{array}{l}\text { "Son espacios de crecimiento } \\
\text { para fortalecer competencias } \\
\text { muy importantes al momento } \\
\text { de desarrollar nuestras } \\
\text { actividades académicas, } \\
\text { súper". }\end{array}$ \\
\hline Estudiante 13 & "Si" & $\begin{array}{l}\text { "Me ayudó a comprender } \\
\text { qué falencias presento y así } \\
\text { aplicarlas en las materias que } \\
\text { estoy viendo". }\end{array}$ \\
\hline
\end{tabular}




\begin{tabular}{|l|c|c|}
\hline PARTICIPANTES & $\begin{array}{c}\text { RESPUESTA } \\
\text { EVALUACIÓN }\end{array}$ & \multicolumn{1}{c|}{ DESCRIPCIÓN } \\
\hline Estudiante 14 & “Si” & $\begin{array}{l}\text { "Son interesantes, despejan } \\
\text { un poco la mente de lo } \\
\text { cotidiano, del estrés y la } \\
\text { carga de cada día, ayudan } \\
\text { a concentrarse mejor, a } \\
\text { observar más detenidamente". }\end{array}$ \\
\hline Estudiante 15 & "No" & $\begin{array}{l}\text { "No tuve tiempo para } \\
\text { dedicarme a esto, veo que son } \\
\text { largas para resolver y no sé } \\
\text { en qué pueden ayudarme" El } \\
\text { estudiante denota completo } \\
\text { desinterés en la estrategia. }\end{array}$ \\
\hline
\end{tabular}

Asimismo, en la entrevista se solicitó al participante evaluar de manera dicotómica las sesiones de entrenamiento frente a su rendimiento académico en el segundo periodo del semestre 2017 mencionando el porqué de sus notas parciales.

Fuente: Elaboración propia.

Tabla II. Entrevistas individuales. Respuesta de la utilidad de las sesiones de entrenamiento vs. promedio académico de los estudiantes en el periodo académico 2017

\begin{tabular}{|c|c|c|c|}
\hline PARTICIPANTE & $\begin{array}{l}\text { RESPUESTA } \\
\text { EVALUACIÓN }\end{array}$ & $\begin{array}{l}\text { PROMEDIO SEMESTRAL } \\
\text { PERIOODO 2017-10 }\end{array}$ & $\begin{array}{l}\text { ASIGNATURAS PERDIDAS } \\
\text { (MOTIVO DE PÉRDIDA) }\end{array}$ \\
\hline Estudiante 1 & “Sí” & 3.8 & Ninguna \\
\hline Estudiante 2 & “Sí” & 3.8 & Física Mecánica con 2.6 por bloqueo en los parciales. \\
\hline Estudiante 3 & “Sí" & 3.56 & Ninguna \\
\hline Estudiante 4 & “Sí" & 3.75 & Ninguna \\
\hline Estudiante 5 & “Sí” & 3.58 & Ninguna \\
\hline Estudiante 6 & “Sí” & 3.62 & $\begin{array}{l}\text { CMD Problemática Ambiental con } 2.7 \text {, el docente solo saca una nota al } \\
\text { corte y es el parcial. }\end{array}$ \\
\hline Estudiante 7 & “No" & 3.05 & Ética Profesional con 1.1 \\
\hline Estudiante 8 & “Sí" & 3.42 & Electiva CMD con 2.6 por retrasos en la llegada. \\
\hline Estudiante 9 & “Sí" & 3.27 & $\begin{array}{l}\text { Probabilidad y Estadística con } 2.5 \text { y Cálculo Diferencial con } 2,4 \text { por } \\
\text { bloqueos en los parciales }\end{array}$ \\
\hline Estudiante 10 & “Sí" & 3.44 & Ninguna \\
\hline Estudiante 11 & “No" & 3.13 & Pavimentos con 1.5 por dificultades en las entregas de trabajos \\
\hline Estudiante 12 & “Sí" & 3.67 & Ninguna \\
\hline Estudiante 13 & "Si" & 2.85 & Pérdidas en cuatro asignaturas por motivos personales y familiares. \\
\hline Estudiante 14 & “Si" & 3.99 & Ninguna \\
\hline Estudiante 15 & “No" & 2.52 & Estructuras de Datos y Cálculo Diferencial \\
\hline
\end{tabular}

Fuente: Elaboración propia.

Tabla III. Comparativo de rendimiento académico periodos 2016-2017. Conglomerado de los promedios académicos de los periodos académicos segundo semestre 2016 y primer semestre 2017

\begin{tabular}{|l|c|c|c|c|c|}
\hline PARTICIPANTE & $\begin{array}{c}\text { RESPUESTA } \\
\text { EVALUACIÓN }\end{array}$ & $\begin{array}{c}\text { PROMEDIO PERIODO } \\
2016-60\end{array}$ & $\begin{array}{c}\text { ASIGNATURAS } \\
\text { PERDIDAS } \\
2016-60\end{array}$ & $\begin{array}{c}\text { PROMEDIO PERIODO } \\
2017-10\end{array}$ & $\begin{array}{c}\text { ASIGNATURAS } \\
\text { PERDIDAS } \\
2017-10\end{array}$ \\
\hline Estudiante 1 & "Sí" & 3.0 & 3 & 3.8 & 0 \\
\hline Estudiante 2 & "Sí" & 2.99 & 3 & 3.8 & 1 \\
\hline Estudiante 3 & "Sí" & 2.83 & 4 & 3.56 & 0 \\
\hline Estudiante 4 & "Sí" & 3.25 & 3 & 3.75 & 0 \\
\hline Estudiante 5 & "Sí" & 3.26 & 3 & 3.58 & 0 \\
\hline
\end{tabular}




\begin{tabular}{|c|c|c|c|c|c|}
\hline PARTICIPANTE & $\begin{array}{l}\text { RESPUESTA } \\
\text { EVALUACIÓN }\end{array}$ & $\begin{array}{c}\text { PROMEDIO PERÍODO } \\
2016-60\end{array}$ & $\begin{array}{c}\text { ASIGNATURAS } \\
\text { PERDIDAS } \\
2016-60\end{array}$ & $\begin{array}{c}\text { PROMEDIO PERÍODO } \\
2017-10\end{array}$ & $\begin{array}{c}\text { ASIGNATURAS } \\
\text { PERDIDAS } \\
2017-10\end{array}$ \\
\hline Estudiante 6 & “Sí" & 3.55 & 3 & 3.62 & 1 \\
\hline Estudiante 7 & "No" & 2.81 & 3 & 3.05 & 2 \\
\hline Estudiante 8 & “Sí" & 2.76 & 3 & 3.42 & 1 \\
\hline Estudiante 9 & "Sí" & 2.77 & 3 & 3.27 & 2 \\
\hline Estudiante 10 & “Sí" & 3.05 & 3 & 3.44 & 0 \\
\hline Estudiante 11 & "No" & 3.05 & 3 & 3.13 & 1 \\
\hline Estudiante 12 & “Sí" & 3.02 & 3 & 3.67 & 0 \\
\hline Estudiante 13 & “Si" & 2.74 & 3 & 2.85 & 2 \\
\hline Estudiante 14 & "Si" & 2.84 & 5 & 3.99 & 0 \\
\hline Estudiante 15 & "No" & 2.8 & 3 & 2.52 & 2 \\
\hline
\end{tabular}

Fuente: Elaboración propia.

Según los resultados evidenciados, doce de quince estudiantes afirman que esta es una estrategia útil porque promueve competencias analíticas, críticas y creativas en el ámbito académico, encontrando así otras formas de solucionar fuera del pensamiento lineal.

A su vez, los entrenamientos propician cambios en la forma en que los estudiantes se relacionan con el entorno académico, el entrenamiento de habilidades básicas en la etapa universitaria hace que estas se sigan entrenando de forma interactiva, interesante y cercana a los estudiantes.

De acuerdo el análisis cualitativo de las entrevistas aplicadas a los estudiantes, se considera que los participantes se toman el tiempo para la ejecución y posterior retroalimentación, adicional a esto permite la apertura a nuevos espacios de aprendizaje, proporcionando no solo la consolidación de información referente al programa que pertenece, sino también propicia en ellos relaciones sociales provechosas para la academia.

Al observar al participante y conversar con él se puede determinar que presta más atención a los detalles, es más observador con el ambiente que lo rodea, algunos participantes mencionan que estas habilidades se fortalecieron debido a que extrapolan a la vida cotidiana haciendo útil el entrenamiento, esto propicia en los estudiantes nuevas formas de explorar actividades, por ejemplo, ellos buscan aplicaciones electrónicas para el desarrollo de las mismas, descargan apps que permitan fortalecer habilidades.

El estudiante evidencia una necesidad de fortalecimiento de las habilidades y por esto toman la iniciativa de retomar la lectura como hábito, buscar espacios para llenar laberintos, sudokus, parqués, entre otros juegos que, a simple vista, son sencillos y cotidianos, y, sin embargo, fortalecen las habilidades mientras se divierten, dan un mejor manejo del tiempo libre o de ocio en actividades sanas y asimismo ayudan al estudiante para que se relacione de una manera amena.

Desde el área educativa de M.A.I.E. se concedió una retroalimentación individual integral de todas las habilidades que el estudiante entrenó desde las retroalimentaciones por correo electrónico como recomendaciones y sugerencias que apuntan al éxito académico, para mejora y practicando constantemente las habilidades cognitivas. Se brindan posibles estrategias para la mejora y perfeccionamiento de las mismas, igualmente, el estudiante se siente relevante para M.A.I.E, lo que los motiva a participar de actividades orientadas por la universidad como también a la permanencia dentro de la institución de educación superior.

Por otro lado, la virtualidad impacta y, además, se les facilita a los estudiantes, puesto que de 250 estudiantes, 235 resolvieron los talleres virtuales, solo 15 asistieron a las sesiones presenciales, teniendo en cuenta que para ambas modalidades se utilizaron las mismas estrategias de divulgación de la información.

Según los resultados encontrados en la investigación, se da respuesta a la pregunta inicialmente planteada y, en concordancia con los resultados expuestos, el juego es una estrategia que aporta al desarrollo de habilidades cognitivas para mejorar el rendimiento académico de los estudiantes con 
pérdida académica de la Facultad de Ingeniería. El juego es una estrategia innovadora, sencilla, y aunque es común en muchos aspectos, fortalece habilidades cognitivas de una manera divertida y provechosa, dando espacios de comunicación, relajación e interacción con sus pares. En este sentido, es importante resaltar que se evidenció que el manejo del tiempo libre no es el adecuado para los estudiantes, lo que conlleva a que la universidad cree espacios dentro del campus para el ocio y la entretención con el objetivo de desarrollar habilidades cognitivas y prevenir problemáticas constantes dentro de este contexto.

Para próximas investigaciones o fases futuras de esta misma, se tendrán en consideración variables como el tiempo de los estudiantes, divulgación por medios audiovisuales y redes sociales como espacios propicios para las actividades y la oferta de entrenamientos en varios días de la semana, con el fin que los estudiantes participen. Por otro lado, se debe realizar la validación de las sesiones de entrenamiento virtuales para dar una mejor retroalimentación al estudiante, tener una estandarización para el mejor desarrollo de habilidades y poder medir qué tanto debe mejorar el estudiante frente a las habilidades trabajadas.

Por otro lado, a partir de las sesiones I, II y III. Se crearon las sesiones virtuales "experto", lo cual permite reforzar la habilidad con las cuales tienen más dificultad (memoria, atención, observación, razonamiento lógico, comprensión lectora). Esta sesión experto tiene mayor exigencia y más dedicación por parte del estudiante.

Adicionalmente, en las sesiones presenciales se conduce la sana competitividad, se retan a ser los mejores, se ven con mayor claridad los errores que usualmente cometían al momento de presentar sus trabajos, tareas y parciales, como el no fijarse en detalles mínimos de un ejercicio matemático o no prestar atención a los signos y símbolos de un texto. Según, la opinión de los participantes, allí encuentran mayor diversión, mayor interacción y mejor comunicación, con ello; se concientiza al estudiante de que las habilidades se fortalecen con otro tipo de estrategias y en otros contextos no solo el educativo.

Por otro lado, se han evidenciado las siguientes problemáticas que desde una escala de autopercepción al $40 \%$ de los estudiantes se les dificulta desarrollar ejercicios que conforman la habilidad de razonamiento lógico y comprensión de lectura; al 33,8\% resolver actividades que implican la observación; al $25,5 \%$ responder el apartado de atención y al $12,4 \%$ ejercicios de memoria. Es por esto que, a raíz de esta investigación. Se han originado nuevas ideas y propuestas como las sesiones de entrenamiento experto, donde no solo se practican sesiones de habilidades mixtas, sino que también se dan sesiones exclusivamente para la habilidad de mayor dificultad, según el caso particular de los estudiantes.

\section{CONCLUSIONES}

Los estudiantes con pérdidas académicas evidencian frecuentemente los errores comunes como el olvido, la falta de atención y la baja comprensión lectora, aunque tengan conocimientos sólidos de los temas propuestos en clase y propios de su programa académico, la carencia o bajo fortalecimiento de las habilidades cognitivas propician una pérdida académica. A la luz de los resultados expuestos en esta investigación las sesiones de entrenamiento en habilidades son útiles para la mejora de su rendimiento académico y su proceso cognitivo. Cabe destacar que este fortalecimiento es sistemático y debe crear el hábito para que haya una notable mejoría y experticia en dichas habilidades.

El uso de esta estrategia permite un apoyo para el estudiante, ya que fortalece sus habilidades y mejora proporcionalmente su proceso académico, propiciando participación activa, relaciones amenas con sus pares e igualmente se apropia de los procesos académicos brindados por la universidad.

\section{AGRADECIMIENTOS}

En primera instancia agradezco a la Facultad de Ingeniería y a MAIE por permitir realizar este proyecto, a Luisa Farfán por la participación en la investigación. A los estudiantes que hicieron participación activa de los entrenamientos, finalmente agradezco a Andrea Noriega y a Camilo Romero por la orientación durante la fase de construcción.

\section{REFERENCIAS}

[1] P. Chacón, El juego didáctico como estrategia de enseñanza-aprendizaje ¿cómo crearlo? Caracas. Universidad Pedagógica Experimental Libertador, 2008.

[2] C. Minerva, El juego: una estrategia importante. Venezuela Universidad de los Andes, 2002. 
[3] V. Renobell. V y F. García, Gamificación en la educación: reinventando la rueda. Universidad camilo José cela. Disponible en: http:// dimglobal.net/revistaDIM34/docs/DIMAP34gamificacion.pdf

[4] M. Ferreyra. Avitabile. C. Botero. J. Paz. F. Urzúa. S, Momento decisivo. La educación superior en América Latina y el Caribe. Washington. Grupo Banco Mundial, 2017.

[5] M. Glanzer, El juego en la niñez: un estudio de la cultura lúdica infantil, aportes a la educación inicial. Aique, 2000. Disponible en: https:// books.google.com.co/books?id=VHeQQAAACAAJ\&dq=el+juego $+y+e l+e s t u d i o \& h l=e s \&-$ sa=X\&ved=0ahUKEwini7L3jITQAhXF5CYKHQB5DWUQ6AEIJDAA

[6] https://pdfs.semanticscholar.org/b359/472a402d3d3c5e78875c0686283dfab5b419.pdf

[7] R. Ocampo, S. Naranjo. M. Arboleda. J. Encalada y B. Jiménez, Diseño, desarrollo y validación de un juego serio en educación superior. Un estudio de caso. Universidad Técnica de Machala. Disponible en: http://investigacion. utmachala.edu.ec/proceedings/index.php/ utmach/article/download/231/200/

[8] N. Charlier, M. Ott, B. Remmele y N. Whitton. Not just for children: Game-based learning for older adults. Academic Conferences International Limited, 2012.

[9] C. Papagno, La arquitectura de los recuerdos: cómo funciona la memoria. Italia;Paidós, 2008.

[10] E. Pérez. Martínez. D. Mendoza. V. Olvera. J (2012) "Habilidades mentales primarias y certeza vocacional en alumnos de primer se- mestre de la licenciatura en Derecho". Paraguay.

[11] A. Pérez-Luño, J. Ramón y J. Sánchez, Análisis exploratorio de las variables que condicionan el rendimiento académico. Sevilla: Universidad Pablo de Olavide, 2000.

[12] E. Añaños, Psicología de la atención y de la percepción: guía de estudio y evaluación personalizada. Barcelona Servei de Publicacions de la Universitat Autónoma de Barcelona. 1999. Disponible en: https://books.google.com.co/ books?id=ZYmTaAiWiH8C\&dq=tipos+de+atencion+psicologia\&hl=es\&source=gbs_navlinks_s

[13] Rips. Serna y G. Flórez, (2013, agosto). El razonamiento lógico como requisito Funcional en Ingeniería. Presentado en $11^{\text {th }}$ LACCEI Latin American and Caribbean Conference for Engineering and Technology. "Innovation in Engineering, Technology and Education for Competitiveness and Prosperity". Disponible en: http://www.laccei.org/LACCEI2013-Cancun/ RefereedPapers/RP221.pdf

[14] D. Coon, Psicología. México: Editorial Thomson, 2004.

[15] P. Corbetta, Metodología y técnicas de la investigación social. España: editorial, 2003.

[16] J. Valenzuela y M. Flores (2012). Fundamentos de investigación educativa. Tecnológico de Monterrey Escuela de Graduados en Educación. Disponible en: https://s6f8d95476e64bd09.jimcontent.com/download/version/.../volumen_2.pdf

[17] Bravo. L., García. U., Hernández. H., Ruíz. M La entrevista, un recurso flexible y dinámico. 2013 México. 